\title{
SH2 mutants of c-src that are host dependent for transformation are trans- dominant inhibitors of mouse cell transformation by activated c-src
}

\author{
Hisamaru Hirai ${ }^{1,4}$ and Harold E. Varmus ${ }^{1-3}$ \\ Departments of Microbiology and Immunology ${ }^{1}$ and Biochemistry and Biophysics, ${ }^{2}$, University of California, San Francisco, \\ California 94143 USA
}

\begin{abstract}
The c-src gene encodes a membrane-associated protein-tyrosine kinase, $\mathrm{pp} 60^{\mathrm{c}-\mathrm{src}}$, whose substrates and regulators have not been identified. In an effort to obtain mutants that might assist the search for proteins that interact with pp60 ${ }^{\text {c-src }}$, we have generated by site-directed mutagenesis two alleles of chicken c-src that are host dependent for transformation and inhibit transformation of mouse cells by activated c-src in a trans-dominant manner. These alleles, named $M 6$ and $M 9$, encode nonconservative changes within the highly conserved FLVRES sequence in the src homology-2 (SH2) region of pp60 c-src, as well as an activating change, Y527F, near the carboxyl terminus. M6 and M9 transform chicken embryo fibroblasts (CEF) more efficiently than the parental allele (Y527F c-src), but fail to transform mouse NIH-3T3 cells. The product of M6-src is less stable than activated $\mathrm{pp}^{-0^{-s s c}}$ in NIH-3T3 cells and shows decreased protein tyrosine kinase activity on all tested substrates; the product of $\mathrm{M9}$-src, however, is stable, has a novel pattern of substrate preference for tyrosine phosphorylation in vitro, and induces a pattern of phosphotyrosine-containing proteins in mouse cells that is similar to that induced by Y527F c-src, even though it fails to transform these cells. Both $M 6$ and $M 9$ inhibit transformation of NIH-3T3 cells by activated c-src in a dose-dependent manner, as assayed by resistance of M6or M9-expressing cells to transformation by $\mathrm{Y} 527 \mathrm{~F}$ c-src or by morphological reversion of cells previously transformed by activated c-src following introduction of $M 6$ or $M 9$. When reversion occurs, the concentration of protein encoded by the active allele declines, without change in level of src mRNA or rate of src protein synthesis, implying that the products of M6 and M9, when present at adequate levels, can destabilize transformation-competent src protein. These alleles offer new opportunities for interfering with the actions of src-related genes and for isolating cellular factors required for the functions of those genes.
\end{abstract}

[Key Words: SH2 mutants; pp60 ${ }^{\text {c-src; }}$ M6 and M9 alleles]

Received September 17, 1990; revised version accepted October 17, 1990.

The products of viral and cellular $s r c$ genes, pp $60^{\text {v-src }}$ and pp60 c-src, are membrane-associated, tyrosine-specific protein kinases whose physiologically relevant effectors and targets have not yet been identified (for reviews, see Jove and Hanafusa 1987; Wyke and Stoker 1987). To obtain mutant src proteins that might exhibit altered interactions with other proteins, we and others have recently focused attention upon two components of pp60 $0^{\text {c-src }}$ known as src homology regions 2 and 3 (SH2 and $\mathrm{SH} 3$; Fig. 1A) (for review, see Pawson 1988). These regions reside outside the kinase domain and are highly conserved among the so-called cytoplasmic protein tyrosine $\mathrm{ki}$ nases (those confined to the cytoplasmic face of the plasma membrane); in addition, $\mathrm{SH} 2$ and $\mathrm{SH} 3$ are found

${ }^{3}$ Address correspondence to Department of Microbiology and Immunology, University of California, San Francisco, California 94143 USA. 'Present address: The Third Department of Internal Medicine, Faculty of Medicine, University of Tokyo, Hongo, Tokyo 113, Japan. in several other proteins implicated in signal transduction, including phospholipase $\mathrm{C} \gamma$, GTPase activator protein (GAP), and the product of the crk oncogene (Mayer et al. 1988; Stahl et al. 1988; Vogel et al. 1988).

Mutations affecting the $\mathrm{SH} 2$ - or $\mathrm{SH} 3$-coding regions of src, fps, and $a b l$ genes have demonstrated that these domains can modulate the enzymatic, structural, and biological properties of cytoplasmic protein tyrosine kinases in complex ways (Bryant and Parsons 1982; Cross et al. 1985; Sadowski et al. 1986; DeClue et al. 1987; DeClue and Martin 1989; Franz et al. 1989; Jackson and Baltimore 1989; Verderame et al. 1989; Wang and Parsons 1989; Hirai and Varmus 1990a,b; O'Brien et al. 1990). We have recently described the production of 34 $\mathrm{SH} 2$ and $\mathrm{SH} 3$ amino acid substitution and deletion mutants of an activated form of chicken c-src. These alleles display a wide range of phenotypes when delivered to chicken embryo fibroblasts (CEFs) in retrovirus vectors: 


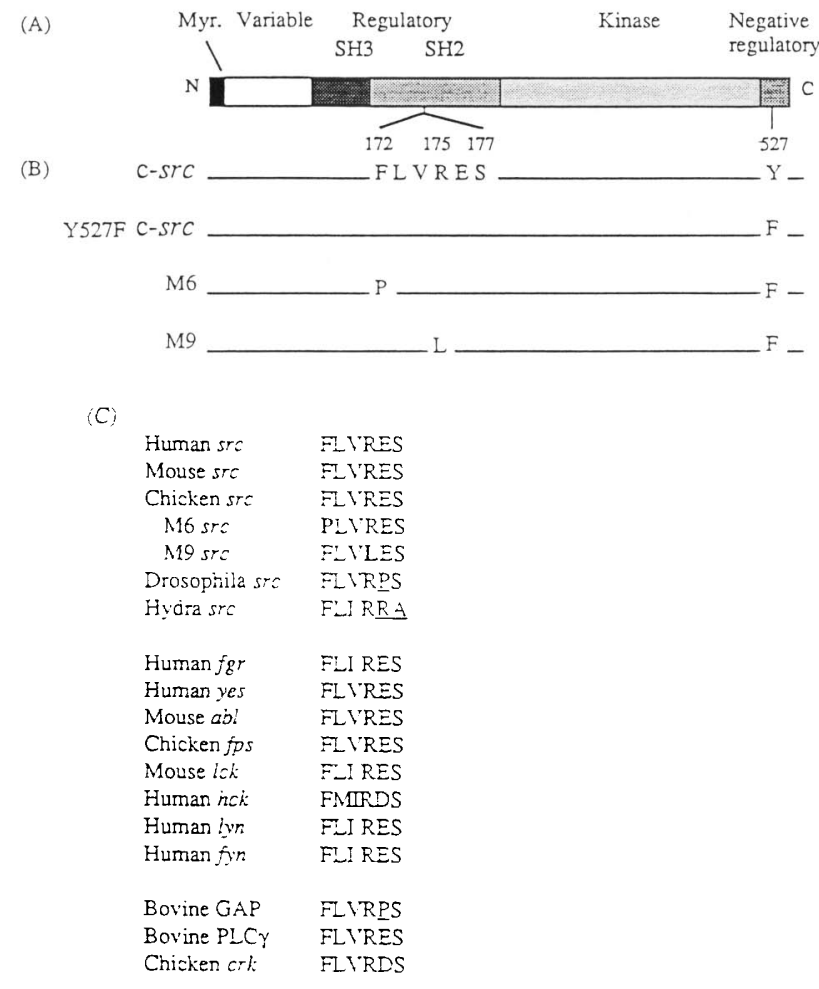

Figure 1. Mutations in the FLVRES sequence of chicken c-src. (A) Functional domains of pp60-src comprising a short aminoterminal sequence required for myristylation $(\mathrm{Myr})$; a region (amino acids 8-88) that varies among src-like protein kinases; the $\mathrm{SH} 3$ and $\mathrm{SH} 2$ domains; the kinase domain; and the carboxy-terminal region containing a site for tyrosine phosphorylation (Y527) that negatively regulates kinase activity of pp60. $(B)$ The four principal alleles of chicken c-src used in this study (normal c-src, Y527F c-src, M6, and M9) and the amino acids at the relevant sites in their predicted products. $|C|$ A compilation of FLVRES-like sequences encoded by the genes of human c-src (Anderson et al. 1985), mouse c-src (Martinez et al. 1987), chicken c-src (Takeya et al. 1983), M6 and M9 src (Hirai and Varmus 1990a), Drosophila c-src (Simon et al. 1985), hydra c-src (Barnekow and Muller 1986), human fgr (Nishizawa et al. 1986), human c-yes (Sukegawa et al. 1987), mouse c-abl (Wang et al. 1984), chicken c-fps (Roebroek et al. 1985), mouse lck (Marth et al. 1985), human hck (Quintrell et al. 1987; Ziegler et al. 19871, human lyn (Yamanashi et al. 1987), human fyn (Kawakami et al. 1986; Semba et al. 1986), bovine GAP (Vogel et al. 1988), bovine phospholipase C (PLC $\gamma$; Stahl et al. 1988), and chicken crk (Mayer et al. 1988; B. Mayer, pers. comm.). Nonconservative differences in naturally occurring genes are underlined, and changes in M6 and M9 srC are $P$ and $L$, respectively.

Some mutant proteins show reduced kinase activity and transforming capacity, others have enhanced activities, and others are unstable (Hirai and Varmus 1990a).

Because earlier reports from our laboratory /Varmus et al. 1981; Verderame et al. 1989) and others (Sadowski et al. 1986; DeClue and Martin 1989/ suggested that some $\mathrm{SH} 2$ mutations are associated with host dependence for transformation, we also introduced many of our mutant alleles into NIH-3T3 mouse fibroblasts with a murine retrovirus vector (Hirai and Varmus 1990b). Several of the $\mathrm{SH} 2$ and $\mathrm{SH} 3$ mutant genes preferentially transformed CEF or NIH-3T3 cells, generally with corresponding differences in kinase activity, implying that the putative regulatory domains influenced the behavior of pp $60^{\text {src }}$ by interacting with host proteins that differed significantly between avian and mammalian host cells.

In our previous survey of mutants, two alleles, called M6 and M9 (Fig. 1B), displayed particularly dramatic host dependence for transformation (Table 1). Both transformed CEFs more efficiently than the parental allele, Y527F c-src; in fact, the activating mutation Y527F was not required for transformation of CEFs when the M6- or M9-specific $\mathrm{SH} 2$ mutations were present in chicken c-src (Hirai and Varmus 1990a). On the other hand, we failed to observe transformation of NIH-3T3 cells with either M6 or M9, even when we selected colonies of infected cells with the highest attainable levels of mutant pp60 (Hirai and Varmus 1990b).

Interestingly, both of these alleles encode nonconservative amino acid changes (F172P or R175L) in the two invariant residues in the FLVRES motif, a sequence extraordinarily well conserved in all $\mathrm{SH} 2$-bearing proteins (Fig. 1C). We have consequently examined the behavior of M6- and M9-encoded proteins more closely. Although both kinases are relatively inactive in vitro when immunoprecipitated from mouse cells, the M9 kinase induces a pattern of phosphotyrosine-containing proteins in NIH-3T3 cells similar to that induced by Y527F c-src, despite its inability to cause neoplastic transformation in these cells; when immunoprecipitated from mouse cells, the M9 kinase exhibits a novel spectrum of substrate preference in vitro. The M6 kinase is relatively inactive on all substrates when synthesized in mouse cells, although both M6 and M9 kinases are more active than that encoded by $\mathrm{Y} 527 \mathrm{~F}$ c-src when produced in CEFs. Moreover, both M6 and M9 can interfere with transformation of NIH-3T3 cells by activated c-src in a dose-dependent manner in trans. Inhibition of transformation is accompanied by destabilization of transformation-competent src protein. Thus, simple alterations in the FLVRES sequence can dramatically affect the behavior of $S T C$ protein in different cellular environments, providing materials for novel approaches to the functions of the src gene family.

\section{Results}

Proteins encoded by M6 and M9 behave differently in avian and mammalian host cells

To assess the basis of the host dependence of the M6 and M9 alleles for transformation, we performed a direct comparison of the proteins encoded by these two mutants, by the activated parental allele (Y527F c-src), and by normal chicken c-src, in CEF and NIH-3T3 cells infected by avian and murine retrovirus vectors (Fig. 2; Table 1). As reported in our earlier surveys, the $\left[{ }^{35} \mathrm{~S}\right] \mathrm{me}$ thionine-labeled proteins encoded by both M6 and M9 accumulate in CEFs to higher concentrations in a 12-hr labeling period than do those encoded by normal and activated c-src (Fig. 2A, left), apparently reflecting prolonged half-lives (Hirai and Varmus 1990a). In NIH-3T3 
Table 1. Biological and biochemical activities of M6 and M9 alleles of c-src

\begin{tabular}{|c|c|c|c|c|c|c|c|c|c|c|c|c|c|c|}
\hline \multirow{4}{*}{$\begin{array}{c}\text { src allele } \\
\text { in } \\
\text { virus vector }\end{array}$} & \multicolumn{4}{|c|}{ Transforming activity ${ }^{\mathbf{a}}$} & & & & & & & & & & \\
\hline & \multirow{2}{*}{\multicolumn{2}{|c|}{$\begin{array}{l}\text { focus- } \\
\text { forming } \\
\text { activity }\end{array}$}} & \multirow{2}{*}{\multicolumn{2}{|c|}{$\begin{array}{c}\text { colonies } \\
\text { in } \\
\text { soft agar }\end{array}$}} & \multirow{2}{*}{\multicolumn{2}{|c|}{ pp60 level ${ }^{b}$}} & \multicolumn{8}{|c|}{ Specific kinase activity ${ }^{c}$} \\
\hline & & & & & & & \multicolumn{2}{|c|}{ enolase } & \multicolumn{2}{|c|}{ angiotensin } & \multicolumn{2}{|c|}{ casein } & \multicolumn{2}{|c|}{ poly(Glu-Tyr) } \\
\hline & $\overline{\mathrm{CEF}}$ & $\overline{3 \mathrm{~T} 3}$ & $\overline{\mathrm{CEF}}$ & $\overline{3 T 3}$ & CEF & $3 \mathrm{~T} 3$ & $\overline{\mathrm{CEF}}$ & $3 \mathrm{T3}$ & CEF & $\overline{3 T 3}$ & $\overline{\mathrm{CEF}}$ & $3 \mathrm{~T} 3$ & CEF & $3 \mathrm{~T} 3$ \\
\hline None & $<0.1$ & $<0.1$ & $<0.1$ & $<0.1$ & $<0.1$ & $<0.1$ & $<0.1$ & $<0.1$ & $<0.1$ & $<0.1$ & $<0.1$ & $<0.1$ & $<0.1$ & $<0.1$ \\
\hline c-src & $<0.1$ & $<0.1$ & $<0.1$ & $<0.1$ & 2.1 & 2.0 & 0.1 & 0.1 & 0.2 & 0.2 & 0.2 & 0.2 & 0.1 & 0.1 \\
\hline Y $527 \mathrm{~F} \mathrm{c}$-src & 1.0 & 1.0 & 1.0 & 1.0 & 1.0 & 1.0 & 1.0 & 1.0 & 1.0 & 1.0 & 1.0 & 1.0 & 1.0 & 1.0 \\
\hline M6 & 1.9 & $<0.1$ & 1.7 & $<0.1$ & 2.6 & 0.3 & 1.8 & 0.3 & 1.5 & 0.2 & 1.7 & 0.3 & 2.0 & 0.2 \\
\hline M9 & 1.5 & $<0.1$ & 2.1 & $<0.1$ & 4.0 & 0.9 & 1.2 & 0.2 & 1.4 & 0.5 & 1.3 & 0.7 & 1.6 & 1.1 \\
\hline
\end{tabular}

RSV and MLV-neo vectors carrying the indicated alleles of chicken c-src were used to infect CEF and NIH-3T3 cells, respectively, as described in Materials and methods.

anfected cells were tested for transformation competence by focus formation and soft agar cloning assays as described previously (Hirai and Varmus 1990b). Results for these and other properties were normalized to 1.0 for the activated allele Y527F c-src.

bLevels of pp60 src were determined as shown in Fig. 2.

cThe specific kinase activities on the four indicated substrates were determined in immunoprecipitates formed with monoclonal antibody 327 as described in Materials and methods. Results of the transformation assays were reported previously (Hirai and Varmus $1990 \mathrm{a}, \mathrm{b})$ and are included here for comparison with the biochemical results, some of which are derived from repetition of experiments described in earlier papers.

cells, however, the M6 protein was less abundant and the $\mathrm{M} 9$ protein was no more abundant than protein encoded by Y527F c-src (Fig. 2A, right; Hirai and Varmus 1990b). (Similar results were obtained by the immunoblot procedure with src-specific antiserum; data not shown.)

As measured in a conventional immune complex assay with acid-treated enolase or pp60, itself, as substrates, the kinase activities of the mutant proteins also exhibited host dependence (Fig. 2B; Hirai and Varmus 1990a,b). Thus, both M6 and M9 proteins showed higher specific activities than did pp60 $6527 \mathrm{~F}$ c-src when immunoprecipitated from CEFs, but much lower specific activities when precipitated from NIH-3T3 cells (Table 1).

The M9 kinase made in mouse cells has unusual substrate specificity in vitro and induces abundant phosphotyrosine

To explore the apparent deficiencies in specific kinase activity of M6 and M9 proteins synthesized in mouse cells, we used additional substrates [enolase, angiotensin, casein, and poly(Glu-Tyr)] in the in vitro kinase assay (Table 1) and examined the pattern of phosphotyrosine-containing proteins in cells expressing the mutant alleles (Fig. 3).

When produced in CEF, the M6 kinase shows a 1.5- to 2-fold higher specific activity than does the product of activated c-src on all four substrates tested; when made in NIH-3T3 cells, however, the specific activity is reduced three- to fivefold on all four substrates (Table 1). The M9 kinase is also slightly more active than activated c-src protein on all substrates after synthesis in CEFs. The M9 kinase from mouse cells is more selective in the use of substrates, showing strongly diminished activity with enolase (see Fig. 2B), twofold reduction with angiotensin, $30 \%$ reduction with casein, and slightly increased activity when compared with pp $60^{\mathrm{Y} 527 \mathrm{~F}} \mathrm{c}-s \mathrm{rc}$ on poly(Glu-Tyr).
As another measure of the kinase activity of the proteins encoded by $\mathrm{M} 6$ and $\mathrm{M} 9$, we used the immunoblot procedure with anti-phosphotyrosine antibodies to $\mathrm{ex}$ amine the abundance of phosphotyrosine-containing proteins in lysates of CEF and NIH-3T3 cells expressing these and control alleles (Fig. 3). As anticipated from results shown in Table 1 and Figure 2, both M6 and M9 alleles induced in CEFs an intensified version of the same pattern of phosphotyrosine proteins observed in cells transformed by Y527F c-src. Also, as expected from the low concentration and specific kinase activity of $M 6$ protein in NIH-3T3 cells, little or no increase in phophotyrosine-containing proteins was observed when mouse cells expressing M6 were compared with cells expressing normal c-src. Surprisingly, however, the M9 allele induced a pattern of phosphotyrosine proteins in NIH-3T3 cells with only a few differences from that induced by the transforming allele, Y527F c-src. Similar findings were observed with three anti-phosphotyrosine antibodies and with multiple clones and mass cultures of NIH-3T3 cells expressing M9-src or Y52F c-src, but we have not yet established the identities of those proteins that account for the few differences in the two patterns. However, the phosphotyrosine-containing proteins detected in $\mathrm{M} 9$-expressing NIH-3T3 cells are apparently not sufficient for transformation, although we cannot exclude a requirement for them.

The findings shown in Figure 3 emphasize the unusual nature of the protein encoded by the $\mathrm{M} 9$ allele. Although the M9 protein is more active than pp $60^{\text {Y } 527 \mathrm{~F} \mathrm{c}-s \mathrm{rc}}$ in chicken cells and although it is stably expressed and able to induce widespread tyrosine phosphorylation in mouse cells, it still fails to cause morphological transformation of mouse cells.

M6 and $M 9$ alleles inhibit transformation of $3 T 3$ cells in trans

Since the M6- and M9-specific mutations in the FLVRES 


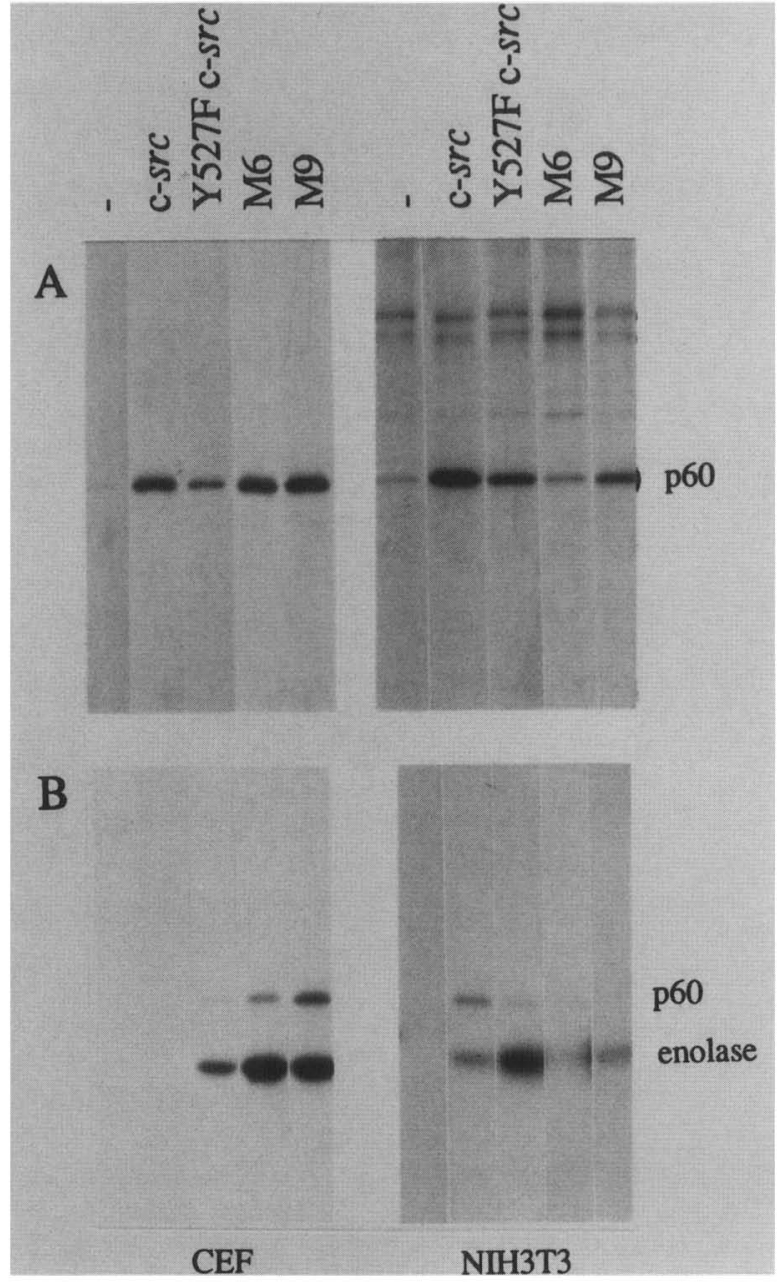

Figure 2. The products of $M 6$ and $M 9$ alleles in CEF and NIH-3T3 cells. $(A)$ The accumulation of $\left[{ }^{35} \mathrm{~S} \mid\right.$ methionine-labeled pp $60^{s t c}$ after $12 \mathrm{hr}$ of metabolic labeling of CEF or G418resistant NIH-3T3 cells infected with RSV or MLV-neo vectors carrying the indicated alleles. Proteins immunoprecipitated from half of each lysate with mAb 2-17 /see Materials and methods) were separated by electrophoresis in a $10 \%$ SDSPAGE. $(B)$ Immunoprecipitates from the remaining half lysate were tested for protein kinase activity by incubation with aciddenatured enolase and $\left[\gamma^{-32} \mathrm{P} \mid\right.$ ATP before SDS-PAGE. The positions of enolase and autophosphorylated pp 60 are indicated. Uninfected cells and c-src- or Y527F c-src-infected cells are included as controls.

sequence inhibited in cis the competence of Y527F c-src to transform NIH-3T3 cells, it seemed possible that the M6 and M9 alleles might also exhibit a trans-dominant effect upon the transformation competence of activated c-src.

Cells expressing M6 or M9 are resistant to transformation We first asked whether the production of M6- or M9-encoded src proteins rendered $\mathrm{NIH}-3 \mathrm{~T} 3$ cells resistant to transformation by the activated c-src allele, Y527 c-src, present in a superinfecting MLV vector (Table 2 ). Clones of NIH-3T3 cells were derived after infection with MLV-gpt vectors carrying the following c-src al- leles; M6, M9, normal c-src, activated c-src, and two nontransforming alleles with deletions encompassing part or all of the FLVRES sequence [residues 93-224 (D14) and residues 166-175 (D6)]. Most clones used for the superinfection experiment contained amounts of pp60 equal to or greater than that determined previously to be just over the threshold required for transformation of NIH-3T3 cells by Y527F c-src (denoted 1.0 in Table 2; see Hirai and Varmus 1990b|. However, the highest observed levels of M6 protein and of protein encoded by the D14 mutant were only about half this value.

Following superinfection with MLV-neo carrying activated c-src, G418-resistant colonies were observed for their morphologic phenotype. From microscopic evaluation of several hundred colonies from each superinfected culture, it was apparent that the cell lines expressing M6 and $\mathrm{M} 9$ were relatively resistant to transformation by activated c-src (Table 2). Whereas MLV carrying activated c-src transformed approximately two-thirds $(60-72 \%)$ of control cells (normal NIH-3T3 cells or cells expressing normal c-src or the two deletion mutants), less than one third $(28-31 \%)$ of the superinfected cells expressing M6 or M9 were transformed. When the experiment was repeated with lines containing two- or threefold higher levels of M9-encoded protein, the resistance to transformation increased slightly, with only $19 \%$ of the G418-resistant colonies being transformed at the highest level. In contrast, cells expressing more normal pp60-src were transformed with equal or slightly higher efficiencies. Superinfection of cells already transformed by Y527F c-src produced, as expected, antibioticresistant colonies that were nearly uniformly transformed.

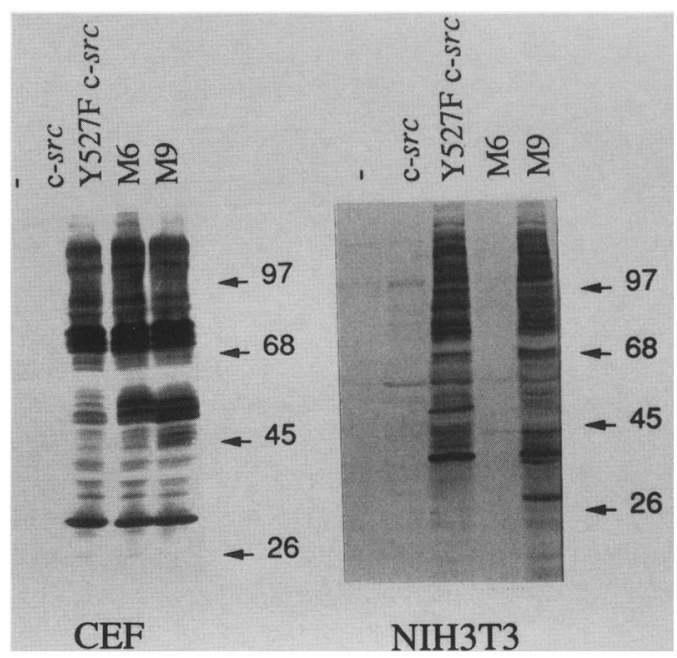

Figure 3. Phosphotyrosine-containing proteins from CEF and $\mathrm{NIH}-3 \mathrm{~T} 3$ cells expressing M6 and M9 alleles. Lysates of uninfected CEF and NIH-3T3 cells and cells infected with RSV or MLV-neo vectors bearing the indicated alleles of c-src were subjected to SDS-PAGE, transferred to nitrocellulose, and probed with affinity-purified, polyclonal anti-phosphotyrosine antibody (provided by J. Wang; see Materials and methods). Uninfected and c-src- or Y527F c-src-infected cells are included as controls. Molecular size standards are indicated in kilodaltons. 
Table 2. M6 and M9 alleles confer resistance to transformation

\begin{tabular}{|c|c|c|c|c|c|c|c|c|}
\hline & & \multicolumn{7}{|c|}{$s r c$ allele present before superinfection } \\
\hline & & & & Y527F & & & & \\
\hline & & none & $c \cdot s r c$ & $c-s r c$ & M6 & M9 & D6 & D14 \\
\hline \multirow[t]{4}{*}{ (A) $0.1 \leqslant p p 60 \leqslant 1$} & pp60 level & 0.1 & 1.0 & 1.0 & 0.5 & 1.0 & 1.0 & 0.5 \\
\hline & neo ${ }^{\mathrm{R}}$ clones & 477 & 449 & 423 & 391 & 416 & 425 & 432 \\
\hline & transformed & 286 & 324 & 408 & 109 & 129 & 298 & 273 \\
\hline & transformed $(\%)$ & 60 & 72 & 96 & 28 & 31 & 70 & 63 \\
\hline \multirow[t]{3}{*}{ (B) $\mathrm{pp} 60=2.0$} & neo $^{R}$ clones & & 380 & 404 & & 387 & 412 & \\
\hline & transformed & & 292 & 402 & & 93 & 327 & \\
\hline & transformed $(\%)$ & & 77 & $>99$ & & 24 & 79 & \\
\hline \multirow[t]{3}{*}{ (C) $\mathrm{pp} 60=3.0$} & neo ${ }^{R}$ clones & & 430 & 415 & & 417 & & \\
\hline & transformed & & 345 & 414 & & 79 & & \\
\hline & transformed $(\%)$ & & 80 & $>99$ & & 19 & & \\
\hline
\end{tabular}

Clones of NIH-3T3 cells infected previously with MLV-gpt vectors carrying the indicated alleles of c-src were superinfected with an MLV-neo vector containing Y527F c-src. The levels of pp60 expressed (relative to a level of protein, 1.0, the minimum sufficient for transformation by $\mathrm{Y} 527 \mathrm{~F} \mathrm{c}-\mathrm{src})$ are listed for each experiment. The indicated number of $\mathrm{G} 418$-resistant $\left(\right.$ neo $^{\mathrm{R}} \mid \mathrm{colonies}$, selected as described in Materials and methods, were scored by microscopy for morphological transformation. The 0.1 pp $60^{s r c}$ level in cells infected with MLV-gpt without a $s r c$ allele (experiment A) was endogenous mouse pp $60^{\mathrm{c}-s r c}$. Codons $166-175$ are deleted from Y527F c-src in the D6 allele, and codons 93-226 are deleted from Y527F c-src in D14.

Introduction of M6 or M9 reverts transformed cells to normal morphology The results of the superinfection experiment presented in Table 2 suggested that the M6and $\mathrm{M} 9$-encoded proteins could prevent transformation by activated c-src in a dose-dependent manner. To corroborate this finding with a different experimental strategy, we asked whether the M6 and M9 alleles could revert the morphological phenotype of cells transformed by activated c-src (Table 3). We therefore superinfected NIH-3T3 lines containing different amounts of pp60 ${ }^{\mathrm{Y} 527 \mathrm{~F}} \mathrm{c}-\mathrm{src}$ with MLV-neo vectors carrying the same set of src alleles tested previously for their ability to confer resistance to transformation. Antibiotic-resistant colonies were then examined for morphologic evidence of partial or complete reversion, as manifested by flattening of the cells and loss of refractility (Fig. 4).

Using a transformed cell line with levels of activated c-src protein just above the threshold value of 1.0 , we observed reversions of $35 \%$ and $40 \%$, respectively, of the colonies obtained after infection with MLV-neo carrying M6 and M9 alleles. In contrast, $<10 \%$ of the colonies in any of the other cultures displayed loss of the transformed phenotype by morphologic criteria, and these changes tended to be less pronounced than in the cells exposed to M6 and M9 viruses. When the experiment was performed with cells containing higher levels of pp $60^{\text {Y527Fc-src }}$, the frequency of reversion was reduced to $17 \%$ and $21 \%$, respectively, with activated c-src protein at the 2.0 level (controls $5 \%$ or less), and to apparently insignificant numbers (both M6 and $M 9$ alleles at $4 \%$ ) at the 3.0 level (controls $2 \%$ or less). Thus, in the reversion test, as well as in the test of resistance to transformation, both the M6 and M9 alleles appeared to interfere with transformation by activated c-src in a dose-dependent fashion. We conclude from these experiments that the M6 and M9 mutants suppress transformation by Y527F c-src in a trans-dominant negative manner.
Inhibition of transformation by M6 or M9 destabilizes protein encoded by a transforming allele of $\mathrm{c}$-src

To define more rigorously the role of src protein concentrations in the observed trans-dominant effects, we repeated the reversion analysis with an activated c-src protein that can be distinguished physically from the products of $M 6$ and $M 9$. In previous experiments we showed that the Dll deletion mutant of Y527F c-src, lacking amino acids 93-143, can transform NIH-3T3 cells with efficiencies similar to those observed for the parental allele, even though it has poor transforming capacity in CEFs, where the protein is relatively unstable (Hirai and Varmus 1990a,b). Because the product of the D11 mutant is $\sim 5 \mathrm{kD}$ smaller than pp $60^{c-s r c}$, it is readily separated from the products of M6 and M9 mutants by polyacrylamide gel electrophoresis (PAGE).

A clone of NIH-3T3 cells transformed by MLV-gpt carrying the D1I allele was superinfected with MLV-neo vectors containing M6 or M9 alleles. In addition, cells expressing M6 or M9 alleles were superinfected with MLV-gpt carrying the D11 allele. Several clones of cells displaying either a persistently transformed or morphologically flat phenotype, like those illustrated in Figure 4 , were isolated and expanded for biochemical tests. Immunoprecipitation after metabolic labeling for $12 \mathrm{hr}$ with ${ }^{35} \mathrm{~S} \mid$ methionine showed that levels of M6 and M9 proteins were modestly but consistently higher in flat than in transformed cells (Fig. 5A). Levels of the active $55-\mathrm{kD}$ protein encoded by the D11 allele, on the other hand, were dramatically decreased in the morphologically normal cell lines. |A variable amount of a smaller protein was also observed in lines expressing M6 or M9; it has not been proved, but is assumed that this is a degradation product of $s r c$ protein, similar to $52-\mathrm{kD}$ product of pp60 $0^{v-s r c}$ described previously (Levinson et al. 1980)].

We performed two kinds of experiments to show 
Table 3. M6 and M9 alleles of $c$-src can revert NIH-3T3 cells transformed by activated $c$-src

\begin{tabular}{|c|c|c|c|c|c|c|c|c|}
\hline & & \multicolumn{7}{|c|}{$s r c$ allele in superinfecting virus } \\
\hline & & \multicolumn{5}{|c|}{$\mathrm{Y} 527 \mathrm{~F}$} & \multirow[b]{2}{*}{ D6 } & \multirow[b]{2}{*}{ D14 } \\
\hline & & none & $\mathrm{c}-\mathrm{src}$ & $\mathrm{c}-\mathrm{SrC}$ & M6 & M9 & & \\
\hline \multirow[t]{5}{*}{ (A) $\mathrm{pp} 60=1.0$} & neo ${ }^{\mathrm{R}}$ clones & 920 & 427 & 398 & 382 & 405 & 411 & 393 \\
\hline & transformed & 860 & 391 & 390 & 227 & 224 & 377 & 367 \\
\hline & partially flat & 28 & 27 & 6 & 89 & 107 & 21 & 17 \\
\hline & & 4 & 12 & 2 & 45 & 57 & 8 & 4 \\
\hline & reverted $\{\%\rangle$ & 3 & 9 & 2 & 35 & 40 & 7 & 5 \\
\hline \multirow[t]{5}{*}{ (B) $\mathrm{pp} 60=2.0$} & neo ${ }^{\mathrm{R}}$ clones & 880 & 452 & 423 & 403 & 391 & 428 & 415 \\
\hline & transformed & 802 & 428 & 420 & 326 & 301 & 409 & 400 \\
\hline & partially flat & 4 & 16 & 2 & 48 & 59 & 14 & 12 \\
\hline & flat & 4 & 8 & 1 & 21 & 23 & 5 & 3 \\
\hline & reverted $(\%)$ & 1 & 5 & 1 & 17 & 21 & 4 & 4 \\
\hline \multirow[t]{5}{*}{ (C) pp60 $=3.0$} & neo $^{\mathrm{R}}$ clones & 960 & 431 & 407 & 377 & 386 & 402 & 423 \\
\hline & transformed & 956 & 420 & 406 & 361 & 369 & 395 & 415 \\
\hline & partially flat & 4 & 8 & 1 & 11 & 10 & 5 & 7 \\
\hline & flat & 0 & 3 & 0 & 5 & 7 & 2 & 1 \\
\hline & reverted $(\%)$ & $<1$ & 2 & $<1$ & 4 & 4 & 2 & 2 \\
\hline
\end{tabular}

NIH-3T3 cells transformed by infection with an MLV-gpt vector carrying Y527F c-src and expressing three levels of pp60/1.0 for experiment A, 2.0 for experiment B, and 3.0 for experiment $\mathrm{Cl}$ were superinfected with MLV-neo vectors carrying the indicated alleles of c-src (see legend to Table 2). G418-resistant colonies were scored by light microscopy for partial or complete restoration of the normal NIH-3T3 morphological phenotype.

whether the unexpectedly low level of D11 src protein in flat cells was caused by regulation of mRNA levels, src protein synthesis, or protein stability. First, we measured steady-state concentrations of src mRNA in transformed and flat cells (Fig. 6). Radiolabeled probes for the bacterial genes (neo and gpt) present in the two MLV vectors distinguished between the vector-length RNAs that serve as mRNAs for D11 protein and for M6 or M9 proteins. [The probes also detect smaller spliced or alternatively initiated mRNAs; these are more abundant from the MLV-neo vector, which contains an internal herpes simplex virus thymidine kinase (HSVtk) promoter.] We observed little or no differences in the abundance of D11 src mRNA in coinfected lines that did and did not display a transformed phenotype. Thus, we cannot explain by changes in mRNA concentration the marked decline in D11 src protein in flat cell lines, whether obtained by reversion or by resistance to transformation. Likewise, similar amounts of M6 and M9 src mRNAs were found in all clones, although we might not have detected the relatively subtle differences required to explain the differences in M6 and M9 protein levels seen in Figure 5A.

To examine the rate of $s r c$ protein synthesis in flat and transformed lines, proteins were labeled with ${ }^{35} S \mid$ methionine for $1 \mathrm{hr}$, rather than $12 \mathrm{hr}$, and subjected to immunoprecipitation and PAGE as before (Fig. 5B). Under these conditions, the amounts of labeled D 11 protein are very similar in cell lines with either phenotype, indicating that altered rates of synthesis do not account for depressed accumulation during a prolonged labeling period. As expected, the rates of synthesis of M6 and M9 proteins also appear nearly invariant among the lines. We conclude that the reduction in the active D11 protein can be ascribed to protein instability in the presence of a critical level of M6 and M9 protein and that reduced levels of the transformation-competent protein contribute to the dominant-negative effect.

\section{Discussion}

Amino acid substitutions in an especially well-conserved portion of the SH2 domain of a transformationcompetent mutant of chicken pp60 ${ }^{c-s s c}$ are encoded by alleles M6 and M9 that are host dependent for transformation and capable of inhibiting transformation of $\mathrm{NIH}-3 \mathrm{~T} 3$ cells by activated c-src. It is striking that the proteins encoded by M6 and M9 behave differently, both biochemically and biologically, in the environments of $\mathrm{CEF}$ and in an established line of mouse fibroblasts and interfere in trans with the oncogenic function and stability of a nearly identical transforming protein in the mouse cells. Indeed, it seems very likely that the phenomena of host dependence and dominant-negative behavior are closely related, perhaps mediated through interactions between the src gene products and cellular protein|s|.

The FLVRES motif that has been altered by mutation in the M6 and M9 alleles does not vary at all between mammalian and avian versions of c-src (Fig. 1C), and there are few amino acid differences throughout the $\mathrm{SH} 2$ regions; hence, the greater activity exhibited in CEF by the products of these alleles cannot be attributed simply to their avian origin. This is supported by studies of other alleles of chicken c-src, most with mutations in SH3, that encode proteins more active in mouse NIH-3T3 cells than in chicken embryo cells (Hirai and Varmus 1990b). (The D11 mutant used for the experiment shown in Figs. 5 and 6 is an example of such a host-dependent allele.) Nevertheless, it will be of ob- 


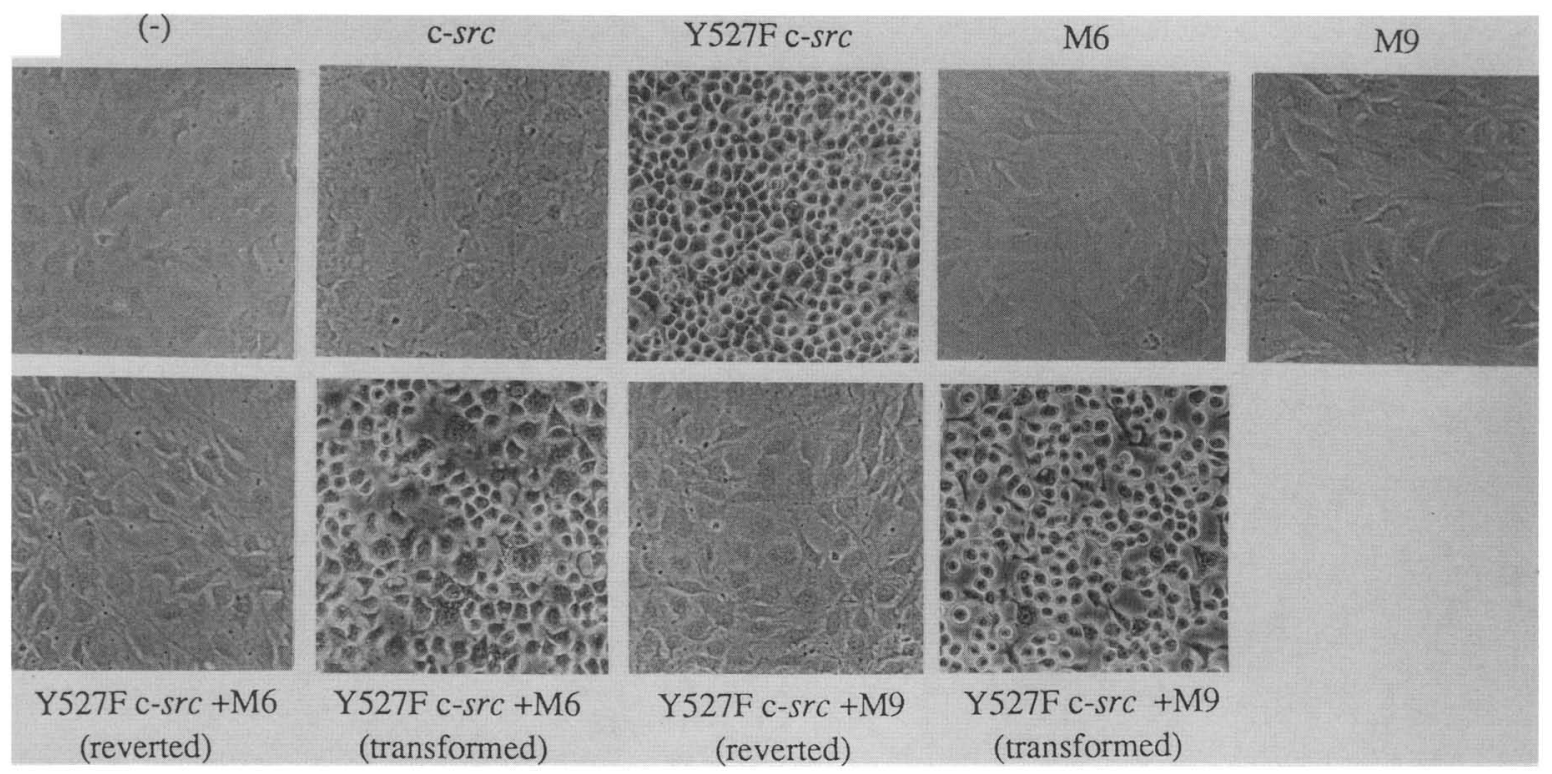

Figure 4. M6 and M9 alleles can revert the phenotype of NIH-3T3 cells transformed by Y527F c-src. MLV-neo vectors carrying M6 or M9 were used to infect $\mathrm{NIH}-3 \mathrm{~T} 3$ cells transformed by threshold levels of pp $60^{\mathrm{Y} 527 \mathrm{Fc}-s t \mathrm{c}}$. After selection with G418, colonies with reverted and persistently transformed phenotypes were photographed under light microscopy at $100 \times(b o t$ tom $)$. As controls, uninfected cells and colonies of cells infected with the indicated c-src alleles are also shown (top). (For further details, see Table 3.)

vious interest to make lesions that are analogs of those in M6 and M9 in mammalian c-src genes to look for host-dependent effects.

We have generated and initially tested all of our SH2 and $\mathrm{SH} 3$ mutants in the background of a c-src gene activated by the Y527F mutation, and we have yet to ask whether the mutation at position 527, normally a site for an inhibitory tyrosine phosphorylation, is required for the trans-dominant effect. Similarly, we do not know which regions of the proteins encoded by M6 and M9 must be retained for this effect; additional mutations that remove the variable, $\mathrm{SH} 3$, and kinase regions or more precisely, inactivate the catalytic function of M6or M9-encoded pp60, will need to be tested to address this question. Although preliminary experiments $(\mathrm{H}$. Hirai, unpubl.) indicate that the M6 and M9 proteins are associated with membranous components of NIH-3T3 cells, more rigorous localization of the proteins and mutagenesis to eliminate membrane association will be required to judge the effects of the intracellular position of pp60 on the phenotypes that we have observed.

Reversion and prevention of Y527F c-src-mediated transformation by $\mathrm{M} 6$ and $\mathrm{M} 9$ are incomplete and dose dependent, suggesting that a critical ratio of the products of the dominant-negative and activated allele is necessary for the nontransformed phenotype (Tables 2 and 3 ) and for destabilization of the transforming protein (Figs. 5 and 6). This result is consistent with the idea that two closely related proteins are competing for some limited host factor essential for transformation by src. However, we have yet to examine carefully the possibility that the M6 and M9 alleles can interfere with c-src functions other than transformation by the Y527F allele, with other oncogenes or proto-oncogenes in the src gene family, or with other proteins containing SH2 domains. We have thus far been unable to revert the phenotype of NIH-3T3 cell lines transformed by v-src, polyoma middle $\mathrm{T}$ antigen, or $\mathrm{v}$-Ha-ras by superinfection with vectors carrying M6 or M9 (H. Hirai, unpubl.), but we cannot say whether these failures should be attributed to the relative strengths of these oncogenes /compared with Y527F c-srcl, to unfavorable ratios of mutant to transforming proteins in the cell lines, or to an inherent inability of the M6 or M9 genes to act as dominant-negative alleles in these settings.

Although M6 and M9 are the first alleles of src to display dominant-negative phenotypes, several oncogenes and related genes have recently been reported to have a variety of trans-inhibitory effects. (1) Most closely related to the phenomena described here is the dose-dependent inhibition of $\mathrm{p} 21^{\mathrm{v} \text {-Ki-ras }}$-mediated transformation of rat cells by overexpression of a human cDNA clone encoding the $21-\mathrm{kD}$ product of $\mathrm{Krev}-\mathrm{l}$ (raplA), a gene belonging to the ras family (Kitayama et al. 1989). (2) Inherited alleles of the mouse c-kit/W gene with mutations in the kinase domain can produce abnormal phenotypes in the presence of a normal allele (Reith et al. 1990). (3) Mutant alleles of the p53 locus, a tumor suppressor gene, appear to contribute to oncogenesis or transformation by interference with normal p 53 (Finlay et al. 1989). (4) The product of the v-erbA oncogene blocks the normal repressive function of the thyroid hormone-responsive transcription factor encoded by the c-erbA proto-oncogene (Damm et al. 1989).

The explanation of such dominant-negative effects is believed to depend on formation of inactive multimers of mutant and wild-type proteins or on competition between mutant and wild-type proteins for some essential 


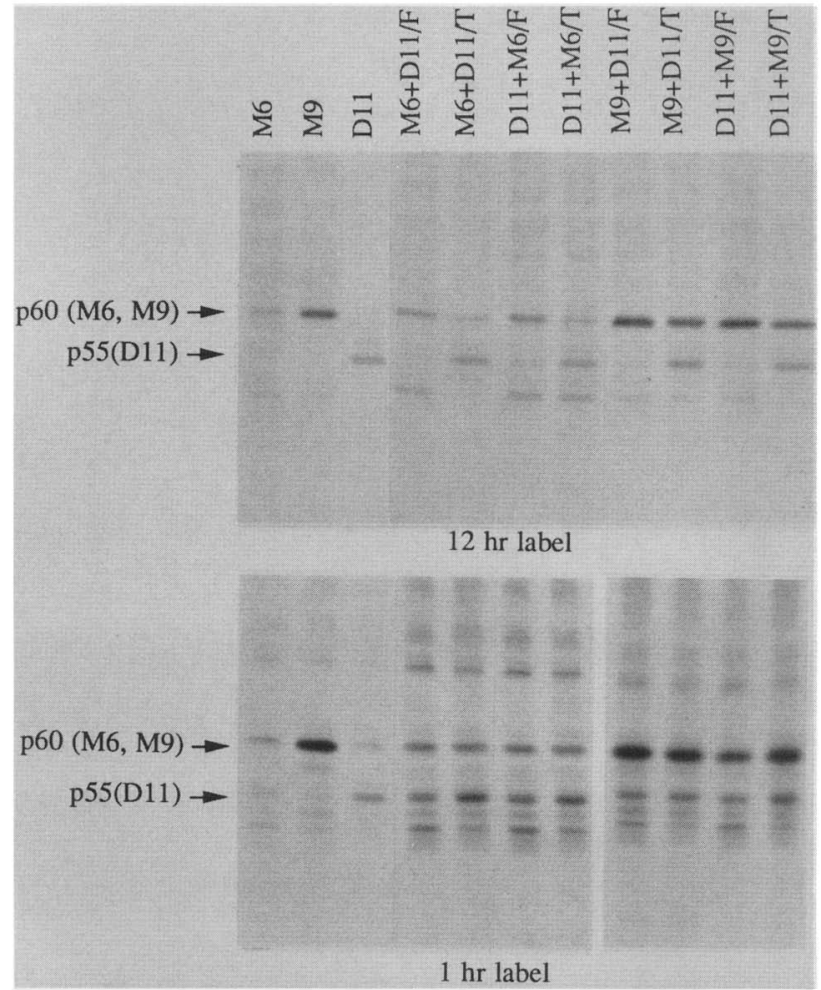

Figure 5. Trans-dominant inhibition of transformation by M6 or M9 alleles destabilizes active c-src protein in NIH-3T3 cells. Experiments analogous to those described in Tables 2 and 3 were performed with the SH3 deletion mutant, D11 (Y527F c-src lacking codons 93-1431, as an active allele in the MLV-gpt vector and with M6 or M9 alleles in the MLV-neo vector. Flat $(\mathrm{F})$ and transformed $(\mathrm{T})$ cells obtained from these experiments were grown to mass culture, metabolically labeled with [ ${ }^{35} \mathrm{~S}$ ]methionine for $12 \mathrm{hr}$ (top) or $1 \mathrm{hr}$ (bottom), and analyzed by immunoprecipitation with mAb 2-17 and SDS-PAGE. Arrows indicate the positions of products of the M6 and M9 alleles (p60) and of the D11 allele (p55). (F) flat cell phenotype; $(T)$ transformed cell phenotype. The order of the alleles over each lane indicates the order in which they were added to produce each cell line.

target (e.g., an enzymatic substrate or binding element; Herskowitz 1987). Thus, the v-erbA and c-erbA proteins may compete for DNA-binding sites in several enhancer elements, and mutant kit proteins may inactivate wildtype proteins when oligomers form in response to physiologic ligands. Because src proteins are believed to remain monomeric in cells (Levinson et al. 1980; Brugge 1986), it is likely that the mechanism of interference involves other proteins that interact with $\mathrm{pp} 60^{\mathrm{src}}$. Many candidates may be considered, including proteins that modify pp $60^{\text {src }}$ (e.g., by phosphorylation or myristylation) or serve as substrates for the protein-tyrosine kinase activity of pp60 src (for review, see Cooper 1990), factors that may localize pp60 $0^{\text {sc }}$ to cell membranes or the cytoskeleton (Resh 1989; Kaplan et al. 1990), proteins (hsp90 and an unidentified protein of $50 \mathrm{kD}$ ) known to form a transient complex with pp $60^{\mathrm{v}-s r c}(\mathrm{Op}$ perman et al. 1981; Brugge 1986), and other proteins in the signal transduction pathway containing $\mathrm{SH} 2$ domains (Stahl et al. 1988; Vogel et al. 1988).

Because it is likely that the dominant-negative and host-dependent effects of M6 and M9 are related, it may be possible to identify the host component for which activated pp $60^{\mathrm{c}-s c}$ and the products of M6 and M9 compete by exploiting the host-dependent properties. For example, the relevant gene(s) could be isolated from NIH-3T3 cells transfected with chicken DNA that permit transformation by M6 or M9. Alternatively, it might be possible to modify the kinase activity of M6 or M9 proteins in vitro with extracts of chicken or mouse cells; factors responsible for such modifications might prove to be important for the dominant-negative effects.

We consider it probable that the avian and mammalian protein(s) responsible for the host-dependent and dominant-negative properties of the M6 and M9 alleles are homologous proteins that normally interact with $s r c$ (and perhaps src-related) proteins to stabilize them and influence their specific kinase activities. Although src proteins with a normal $\mathrm{SH} 2$ domain do not usually differentiate between the avian and mammalian homologs of the hypothetical protein(s), the FLVRES mutants encoded by M6 and M9 are able to make this subtle dis-

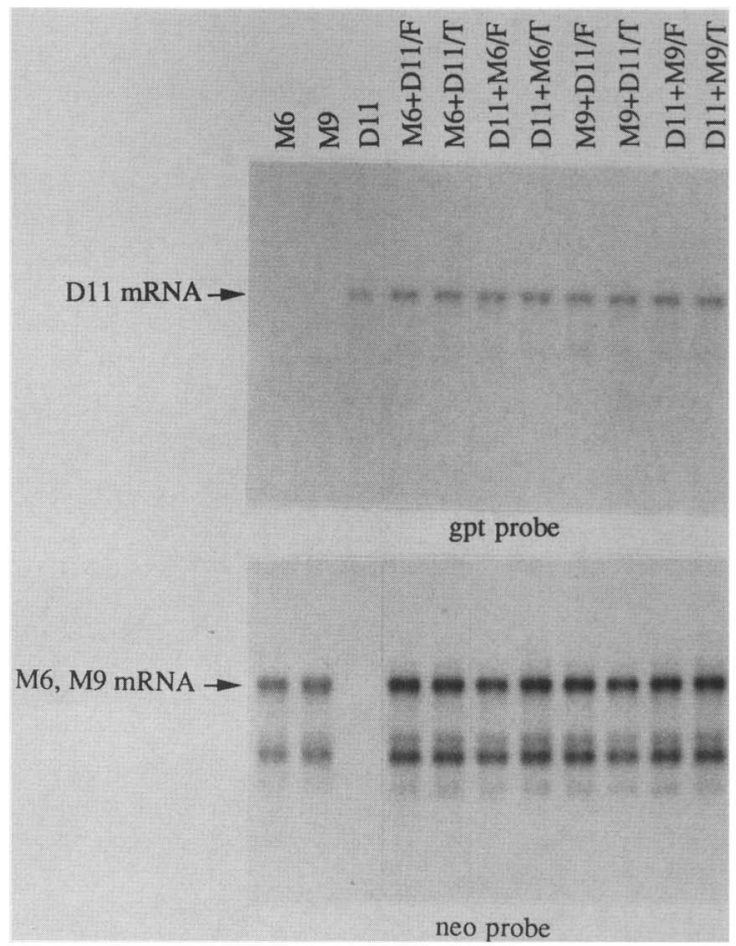

Figure 6. Reversion of transformation by $M 6$ or $M 9$ alleles does not alter levels of src mRNA encoding a transformationcompetent protein. Whole-cell polyadenylated RNA was prepared from the cell lines described in the legend to Fig. 5, subjected to electrophoresis in agarose gels, and probed for species containing D11-src with a ${ }^{32}$ P-labeled probe for Escherichia coli gpt (top) and for species containing M6-and M9-src with a probe for neo $\mathrm{R}^{\mathrm{R}}$ (bottom); arrows indicate the expected positions of the relevant RNAs. $[F \mid$ Flat cell phenotype; $(T)$ transformed cell phenotype. 
crimination. According to this view, M6 and M9 proteins should interact with the mammalian homolog, but the resulting kinase activity is either low (in the case of M6) or altered with respect to substrate specificity (in the case of M91. Presumably, successful competition for the homolog by M6- or M9-encoded proteins can displace the product of Y527F c-src and thereby account for its instability under conditions that produce dominantnegative effects.

\section{Materials and methods}

\section{Plasmids}

M6 (F172P/Y527F c-src) and M9 (R175L/Y527F c-src) mutations were generated by site-directed mutagenesis as described (Kunkel 1985). Normal c-src, Y527F c-src, D6 (del 166-175/ Y527F c-src), D11 (del 93-143/Y527F c-src), and D14 (del 93-226/Y527F c-src) were also used in the analyses. All alleles were inserted into a replication-competent Rous sarcoma virus (RSV) vector (RCAS) for infection of CEFs and MLV-derived retroviral vectors, ZAS, carrying a gpt marker gene (MLV-gpt), and MV7, carrying a neo gene (MLV-neol, for infection of NIH-3T3 cells (Kunkel 1985; Housey 1988; Hirai and Varmus 1990a,b).

\section{Transfection and biological analysis}

Transfection and biological assays for CEFs were performed as described (Hirai and Varmus 1990a). ZAS or MV7 plasmid DNAs were transfected into $\Psi 2$ packaging cells, with or without pSV2-neo DNA, respectively, and transfected cells were selected with medium containing $400 \mu \mathrm{g} / \mathrm{ml}$ of G418 (GIBCO). Culture media were harvested as virus stocks. NIH-3T3 cells were infected with these viruses and selected with gpt-selective medium containing mycophenolic acid $(20$ $\mu \mathrm{g} / \mathrm{ml})$, xanthine $(250 \mu \mathrm{g} / \mathrm{ml})$, and hypoxanthine $(14 \mu \mathrm{g} / \mathrm{ml})$ for MLV-gpt vectors or with G418-containing medium for MLVneo vectors. Biological assays for infected or uninfected $\mathrm{NIH}-3 \mathrm{~T} 3$ cells were described previously (Hirai and Varmus 1990b|. For superinfection experiments, some cell clones expressing an indicated level of mutant src protein were infected with neo viruses, and transformed or flat colonies were scored in medium with G418. p60 levels were determined relative to the threshold level for transformation by parental Y527F c-src (arbitrarily defined as 1.0; Hirai and Varmus 1990b).

\section{Immunoprecipitation and 060 level}

Fully infected CEFs and infected and selected NIH-3T3 cells were labeled with ${ }^{35} \mathrm{~S} /$ methionine in $2 \mathrm{ml}$ of methionine-free, Dulbecco-modified Eagle medium containing $10 \%$ dialyzed fetal calf serum and $400 \mu \mathrm{Ci}$ of $\left[{ }^{35} \mathrm{~S}\right]$ methionine. Labeled cells were lysed in RIPA buffer $[150 \mu \mathrm{M} \mathrm{NaCl}, 20 \mu \mathrm{M}$ Tris $(\mathrm{pH} 7.2)$, $0.1 \%$ sodium dodecyl sulfate (SDS), $1 \%$ sodium deoxycholate, $1 \%$ Triton $\mathrm{X}-100$ ]. Labeling efficiency was determined from the ratio of trichloroacetic acid (TCA)-precipitable radioactivity and total cell protein in each lysate (Kmiecik et al. 1987). Immunoprecipitation of $60^{\mathrm{c}-s r c}$ from lysates containing equal amounts of TCA-precipitable radioactivity were performed using an excess of monoclonal antibody (mAb) 2-17 (obtained from Microbiological Associates), which recognizes residues 2-17 of c-src. Immune complexes were adsorbed to formalinfixed Staphylococcus aureus (Pansorbin; Calbiochem-Behring), and labeled proteins in the complexes were resolved by $10 \%$
SDS-PAGE and analyzed by autoradiography. The amounts of p $60^{\text {src }}$ per milligram of total cell protein were determined from the scintillation counts of gel bands and efficiency of metabolic labeling (Kmiecik et al. 1987). The data for levels of $\mathrm{p}^{\circ} 0^{\text {src }}$ were confirmed by immunoblotting analysis with ${ }^{125}$ I-labeled protein A. Lysates from cells were equalized for protein concentration, loaded onto $10 \%$ polyacrylamide gels, and transferred to nitrocellulose. Filters were blocked with milk and probed with $\mathrm{mAb}$ 327 (provided by J. Bruggel, followed by rabbit anti-mouse immunogloblin (DAIKO) and $2 \mu \mathrm{Ci}$ of ${ }^{125} \mathrm{I}$-labeled protein A (38 $\mathrm{mCi} / \mathrm{ml}$; Amershaml, and analyzed by autoradiography.

\section{In vitro protein kinase assay}

Immune complexes from lysates containing equal amounts of TCA-precipitable radioactivity obtained as above were incubated in kinase buffer $\mid 20 \mathrm{~mm}$ Tris (pH 7.2), $10 \mathrm{mM} \mathrm{MgCl}, 0.1 \%$ Triton X-100] for $10 \mathrm{~min}$ at room temperature with $2 \mu \mathrm{g}$ of acid-denatured rabbit muscle enolase (Sigma), $10 \mu \mathrm{g}$ of casein (Sigma), $10 \mu \mathrm{g}$ of poly(Glu-Tyr) (Sigma), or $5 \mu \mathrm{g}$ of angiotensin (Sigma), and $1 \mu \mathrm{Ci}\left[\gamma^{-32} \mathrm{P} \mid \mathrm{ATP}\right.$ (3000 Ci/mmole; Amersham). The amounts of $\left[{ }^{32} \mathrm{P} \mid\right.$ enolase and $\left[{ }^{32} \mathrm{P}\right]$ casein radioactivity were determined by scintillation counting of excised bands after electrophoresis in 10\% SDS-PAGE. For poly (Glu-Tyr) and angiotensin phosphorylation, a part of the kinase reaction mixture was spotted onto DE-81 paper, washed with phosphate buffer, and counted. The specific kinase activities for these exogenous substrates were calculated in conjunction with the determined amount of exogenous pp $60^{s r c}$ after correction for any detectable endogenous pp60 $60^{\mathrm{r}-s r c}$.

\section{In vivo phosphotyrosine analysis}

Lysates from infected CEF cells and selected NIH-3T3 cells were equalized for protein concentration, separated on a $10 \%$ SDS-PAGE, transferred to nitrocellulose, blocked with bovine serum albumin, and then probed with affinity-purified polyclonal antiphosphotyrosine antibody (provided by J. Wang and $M$. White) or monoclonal antiphosphotyrosine antibody (provided by D. Morrison/ followed by alkaline phosphatase-coupled second antibody (Promega).

\section{Northern blotting}

Total RNA was prepared from cells according to the method described by Kaufmann and Sharp (1982), and poly|A) RNA was selected by oligo(dT)-cellulose. One microgram of poly(A) RNA was electrophoresed on a $1.1 \%$ agarose gel and analyzed by Northern hybridization (Church and Gilbert 1984) with ${ }^{32}$ P-labeled gpt DNA $(0.9 \cdot \mathrm{kb}$ XhoI-HindIII fragment) or neo DNA (0.9-kb PstI fragment).

\section{Acknowledgments}

We thank D. Shalloway, H. Hanafusa, and S. Hughes for molecular clones; M. White, J. Wang, and D. Morrison for phosphotyrosine antibodies; and D. Morgan, C. Lowell, and D. Kaplan for their helpful discussions and comments on the manuscript. This work was supported by a grant from the National Institutes of Health; H.H. was supported by a grant from the Leukemia Society of America; H.E.V. is an American Cancer Society Research Professor.

The publication costs of this article were defrayed in part by payment of page charges. This article must therefore be hereby marked "advertisement" in accordance with 18 USC section 1734 solely to indicate this fact. 


\section{References}

Anderson, S.K., C.P. Gibbs, A. Tanaka, H.-J. Kung, and D.J. Fujita. 1985. Human cellular src gene: Nucleotide sequence and derived amino acid sequence of the region coding for the carboxy-terminal two-thirds of pp60-src. Mol. Cell. Biol. 5: $1122-1129$.

Barnekow, A. and W.A. Muller. 1986. An src-related tyrosine kinase activity in the hydroid, Hydractinia. Differentiation 33: $29-33$.

Brugge, J.S. 1986. Interaction of the Rous sarcoma virus protein pp60src with the cellular proteins pp50 and pp90. Curr. Top. Microbiol. Immunol. 123: 1-22.

Bryant, D. and J.T. Parsons. 1982. Site-directed mutagenesis of the src gene of Rous sarcoma virus: Construction and characterization of a deletion mutant temperature sensitive for transformation. I. Virol. 44: 683-691.

Cooper, I. A. 1990. The src family of protein-tyrosine kinases. In Peptides and protein phosphorylation led. B. Kemp. and P.F. Alewood), pp. 85-113. CRC Press.

Cross, F.R., E.A. Garber, and H. Hanafusa. 1985. N-terminal deletions in Rous sarcoma virus p60 $0^{s t c}$ : Effects on tyrosine $\mathrm{ki}$ nase and biological activities and on recombination in tissue culture with the cellular src gene. Mol. Cell. Biol. 5: 27892795

Church, G.M. and W. Gilbert. 1984. Genomic sequencing. Proc. Natl. Acad. Sci. 81: 1991-1995.

Damm, K., C.C. Thompson, and R.M. Evans. 1989. Protein encoded by v-erb A functions as a thyroid-hormone receptor antagonist. Nature 339: 593-597.

DeClue, J.E. and S. Martin. 1989. Linker insertion-deletion mutagenesis of the v-src genes: Isolation of host-and temperature-dependent mutants. I. Virol. 63: 542-554.

DeClue, J.E., I. Sadowski, G.S. Martin, and T. Pawson. 1987. A conserved domain regulates interactions of the $\mathrm{v}$-fps protein-tyrosine kinase with the host cell. Proc. Natl. Acad. Sci. 84: 9064-9068.

Finlay, C.A., P.W. Hinds, and A.J. Levine. 1989. The p53 protooncogene can act as a suppressor of transformation. Cell 57: 1083-1093.

Franz, W.M., P. Berger, and J.Y.J. Wang. 1989. Deletion of an $\mathrm{N}$-terminal regulatory domain of the c-abl tyrosine kinase activates its oncogenic potential. EMBO /. 8: 137-147.

Herskowitz, I. 1987. Functional inactivation of genes by dominant negative mutations. Nature 329: 219-223.

Hirai, H. and H.E. Varmus. 1990a. Site-directed mutagenesis of the SH2- and SH3-coding domains of c-src produces varied phenotypes, including oncogenic activation of p60c-src. Mol. Cell. Biol. 10: 1307-1318.

1990b. SH2 mutations of active c-src often result in host-dependent phenotypes. Proc. Natl. Acad. Sci. 87: $8592-8596$.

Housey, G.M., M.D. Johnson, W.L.W. Hsiao, C.A. O'Brian, I.P. Murphy, P. Kirschmeier, and I.B. Weinstein. 1988. Overproduction of protein kinase $C$ causes disordered growth control in rat fibroblasts. Cell 52: 343-354.

Hughes, S.H., J.J. Greenhouse, C.J. Petropoulos, and P. Sutrave. 1987. Adaptor plasmids simplify the insertion of foreign DNA into helper-independent retroviral vectors. I. Virol. 61: 3004-3012.

Jackson, P. and D. Baltimore. 1989. N-terminal mutations activate the leukemogenic potential of the myristoylated form of c-abl. EMBO I. 8: 449-456.

Jove, R. and H. Hanafusa. 1987. Cell transformation by the viral src oncogenes. Annu. Rev. Cell. Biol. 3: 31-56.

Kaplan, J.M., H.E. Varmus, and J.M. Bishop. 1990. The src pro- tein contains multiple domains for specific attachment to membrane. Mol. Cell. Biol. 10: 1000-1009.

Kaufman, R.J. and P.A. Sharp. 1982. Construction of a modular dihydrofolate reductase cDNA gene: Analysis of signals utilized for efficient expression. Mol. Cell. Biol. 2: 1304-1319.

Kawakami, T., C.Y. Pennington, and K.C. Robbins. 1986. Isolation and oncogenic potential of a novel human src-like gene. Mol. Cell. Biol. 6: 4195-4201.

Kitayama, H., Y. Sugimoto, T. Matsuzaki, Y. Ikawa, and M. Noda. 1989. A ras-related gene with transformation suppressor activity. Cell 56: 77-84.

Kmiecik, T.E. and D. Shalloway. 1987. Activation and suppression of pp $60^{\mathrm{c}-s r c}$ transforming ability by mutation of its primary sites of tyrosine phosphorylation. Cell 49: 65-73.

Kunkel, T.A. 1985. Rapid and efficient site-specific mutagenesis without phenotypic selection. Proc. Natl. Acad. Sci. 82: 488-492.

Levinson, A.D., H. Oppermann, H.E. Varmus, and J.M. Bishop. 1980. The purified protein product of the transforming gene of avian sarcoma virus phosphorylates tyrosine. I. Biol. Chem. 255: 11973-11980.

Marth, J.D., R. Peet, E.G. Krebs, and R.M. Perlmutter. 1985. A lymphocyte-specific protein-tyrosine kinase gene is rearranged and overexpressed in the murine $T$ cell lymphoma LSTRA. Cell 43: 393-404.

Martinez, R., B. Mathey-Prevot, A. Bernards, and D. Baltimore. 1987. Neuronal pp60 $0^{\mathrm{c}-s r c}$ contains a six amino acid insertion relative to its non-neuronal counterpart. Science 237: 411415.

Mayer, B.J., M. Hamaguchi, and H. Hanafusa. 1988. A novel viral oncogene with structural similarity to phospholipase C. Nature 332: 272-275.

Nishizawa, M., K. Semba, M.C. Yoshida, T. Yamamoto, K. Sasaki, and K. Toyoshima. 1986. Structure, expression, and chromosomal location of the human c-fgr gene. Mol. Cell. Biol. 6: 511-517.

O'Brien, M.C., Y. Fukui, and H. Hanafusa. 1990. Activation of the proto-oncogene p60c-src by point mutation in the $\mathrm{SH} 2$ domain. Mol. Cell. Biol. 6: 2855-2862.

Oppermann, H., A.D. Levinson, L. Levintow, H.E. Varmus, J.M. Bishop, and S. Kawai. 1981. Two cellular proteins that immunoprecipitate with the transforming protein of Rous sarcoma virus. Virology 113: 736-751.

Pawson, T. 1988. Non-catalytic domains of cytoplasmic protein-tyrosine kinases: Regulatory elements in signal transduction. Oncogene 3: 491-495.

Quintrell, N., R. Lebo, H.E. Varmus, J.M. Bishop, M.J. Pettenati, M.M. Le Beau, M.O. Diaz, and J.D. Rowley. 1987. Identification of a human gene (hck) that encodes a proteintyrosine kinase and is expressed in murine hemopoietic cells. Mol. Cell. Biol. 7: 2267-2275.

Reith, A.D., R. Rottapel, E. Giddens, C. Brady, L. Forrester, and A. Bernstein. 1990. W mutant mice with mild or severe de velopmental defects contain distinct point mutations in the kinase domain of the c-kit receptor. Genes Dev. 4: 390-400.

Resh, M.D. 1989. Specific and saturable binding of pp60v-src to plasma membranes: Evidence for a myristyl-src receptor Cell 58: $281-286$.

Roebroek, A.J.M., J.A. Schalken, J.S. Verbeek, A.M.W. Van den Ouweland, C. Onnekink, P.J. Bloemers, and W.J.M. Van de Ven. 1985. The structure of the human c-fes/fps proto-oncogene. EMBO /. 4: 2897-2903.

Sadowski, I., J.C. Stone, and T. Pawson. 1986. A noncatalytic domain conserved among cytoplasmic protein-tyrosine $\mathrm{ki}$ nases modifies the kinase function and transforming activity of Fujinami sarcoma virus pl30gas.tps. Mol. Cell. Biol. 
6: $4396-4408$.

Semba, K., M. Nishizawa, N. Miyajima, M.C. Yoshida, J. Sukegawa, Y. Yamanashi, M. Sasaki, T. Yamamoto, and K. Toyoshima. 1986. yes-related proto-oncogene, syn, belongs to the protein-tyrosine kinase family. Proc. Natl. Acad. Sci. 83: $5459-5463$.

Shenoy, S., J.-K. Choi, S. Bagrodia, T.D. Copeland, J.L. Maller, and D. Shalloway. 1989. Purified maturation promoting factor phosphorylates pp60 $60^{\mathrm{c}-s r c}$ at the sites phosphorylated during fibroblast mitosis. Cell 57: 763-774.

Simon, M.A., B. Dress, T. Kornberg, and J.M. Bishop. 1985. The nucleotide sequence and the tissue specific expression of Drosophila c-src. Cell 42: 831-840.

Stahl, M.L., C.R. Ferenz, K.L. Kelleher, R.W. Kriz, and J. Knopf. 1988. Sequence similarity of phospholipase $C$ with the noncatalytic region of src. Nature 332: 269-272.

Sukegawa, J., K. Semba, Y. Yamanashi, M. Nishizawa, N. Miyajima, T. Yamamoto, and K. Toyoshima. 1987. Characterization of cDNA clones for the human c-yes gene. Mol. Cell. Biol. 7: 41-47.

Takeya, T. and H. Hanafusa. 1983. Structure and sequence of the cellular gene homologous to the RSV src gene and the mechanism for generating the transforming virus. Cell 32: $881-890$.

Varmus, H.E., N. Quintrell, and J. Wyke. 1981. Revertants of an ASV-transformed rat cell line have lost the complete provirus or sustained mutations in src. Virology 108: 28-46.

Verderame, M.F., J.M. Kaplan, and H.E. Varmus. 1989. A mutation in $\mathrm{v}$-src that removes a single conserved residue in the $\mathrm{SH}-2$ domain of $\mathrm{pp} 60^{\mathrm{v}-\mathrm{src}}$ restricts transformation in a hostdependent manner. J. Virol. 63: 338-348.

Vogel, V.S., R.A.R. Dixon, M.D. Schaber, R.E. Diehl, M.S. Marshall, E.M. Scolnick, I.S. Sigal, and J.A. Gibbs. 1988. Cloning of bovine GAP and its interaction with oncogenic ras p21. Nature 335: 90-93.

Wang, H.-C. and J.T. Parsons. 1989. Deletions and insertions within an amino-terminal domain of pp60 $0^{\mathrm{v}-s r c}$ inactivate transforming and modulate membrane stability. I. Virol. 63: $291-302$.

Wang, J.Y.J., F. Ledley, S. Goff, R. Lee, Y. Groner, and D. Baltimore. 1984. The mouse c-abl locus: Molecular cloning and characterization. Cell 36: 349-356.

Wyke, J.A. and A.W. Stocker. 1987. Genetic analysis of the form and function of the viral src oncogene product. Biochem. Biophys. Acta 907: 47-69.

Yamanashi, Y., S. Fukushige, K. Semba, J. Sukegawa, N. Miyajima, K. Matsubara, K. Yamamoto, and K. Toyoshima. 1987. The yes-related cellular gene lyn encodes a possible tyrosine kinase similar to p56 lck. Mol. Cell. Biol. 7: 237-243.

Ziegler, S.F., J.D. Marth, D.B. Lewis, and R.M. Perlmutter. 1987. A novel protein tyrosine kinase gene (hck) preferentially expressed in cell of hematopoietic origin. Mol. Cell. Biol. 7: 2276-2285. 


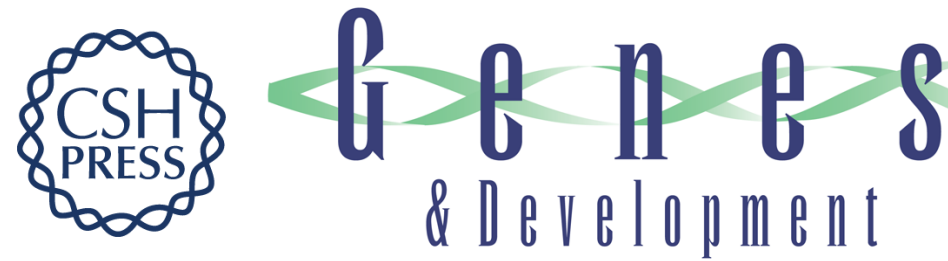

\section{SH2 mutants of c-src that are host dependent for transformation are trans-dominant inhibitors of mouse cell transformation by activated c-src.}

$\mathrm{H}$ Hirai and $\mathrm{H} \mathrm{E}$ Varmus

Genes Dev. 1990, 4:

Access the most recent version at doi:10.1101/gad.4.12b.2342

References This article cites 51 articles, 24 of which can be accessed free at:

http://genesdev.cshlp.org/content/4/12b/2342.full.html\#ref-list-1

License

Email Alerting Service

Receive free email alerts when new articles cite this article - sign up in the box at the top right corner of the article or click here.

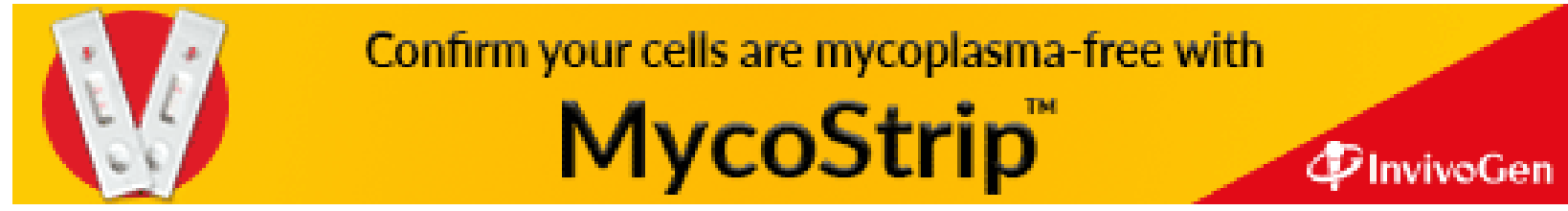

